\title{
Evaluación de dos estimulantes hormonales para el engorde de toretes Brahman bajo pastoreo y bloques multinutricionales suplementarios
}

\author{
Evaluation of two hormonal stimulants for fattening Brahman \\ bulls under grazing and supplementary multinutritional blocks
}

\author{
Eloy Castro ${ }^{1}$, Carmen Pérez ${ }^{1}$, Carlos Alberto Ortega ${ }^{1}$, Diego Salazar Vizuete ${ }^{1}$, Juan Pazmiño González ${ }^{1}$ \\ ${ }^{1}$ Universidad Central del Ecuador. Facultad de Ciencias Agrícolas. Jerónimo Leiton y Av. \\ La Gasca s/n. Ciudadela Universitaria. Quito. 170521. Ecuador
}

\begin{abstract}
Resumen
La alimentación en la producción animal es un reto que enfrentan los agricultores al buscar que el animal alcance el peso ideal en el menor tiempo y al menor costo posible. Se decidió evaluar dos estimulantes hormonales, Fenilpropionato Nandrolona y Boldenone Undecylenate, en combinación con un suministro de mezcla mineral, bloque proteico-energético y pasto miel en el engorde de toretes Brahman comparados con un testigo de manejo del productor. Para el análisis estadístico se utilizó un diseño completamente al azar. Las variables a evaluar fueron incremento de peso, incremento de cinchera y altura a la cruz. El mejor tratamiento en términos de las tres variables evaluadas fue el tratamiento que utilizó anabólico sintético inyectable Undecilenato Boldenona combinado con bloque multinutricional proteico energético mineralizado y vitaminizado, mientras que el testigo tuvo solamente incrementos marginales. El análisis financiero determinó que el mismo tratamiento fue el que presentó la mejor relación beneficio costo de US\$ 1.112 .
\end{abstract}

Palabras clave: alimentación, hormonas, bloque multinutricional, ganancia de peso.

\begin{abstract}
Feeding in animal production is a challenge that farmers face in the search of the ideal animal weight in the least amount of time and at the lower cost. An experiment was designed to evaluate two hormonal stimulants, Phenylpropionate Nandrolone and Boldenone Undecylenate combined with a supply of mineral mix nutrition block and honey grass on Brahman steers compared with a check which was the normal farmer's management. Statistical analysis was performed using a completely randomized design. Variables evaluated were weight gain, cinch increase and height at withers. The best treatment in terms of the three evaluated parameters was the one which used injectable synthetic anabolic Fenilpropionato Nandrolona, while the check only produced marginal gains. Financial analysis determined that the same treatment promoted the best cost-profit of $\$ 1.112$
\end{abstract}

Keywords: feeding, hormones, multinutritional block, weight gain. 


\section{Introducción}

La ganadería bovina aporta significativamente a la alimentación del país. El litoral ecuatoriano produce el $65 \%$ de la carne que se consume, mientras que la Sierra se especializa en la producción de leche y sus derivados. El cantón San Miguel de los Bancos situado al noroccidente de la provincia de Pichincha cuenta con las condiciones climáticas que permiten el desarrollo de una diversidad de pastizales que se transforman en leche con variados porcentajes de proteína y en cárnicos con parámetros de producción y productividad considerados bajos. Según el último censo agropecuario, el promedio de la tasa de crecimiento de carne es de 286 g/día, la carga animal es de solamente 0,8 Unidades Bovinas Adultas (UBA)/ha/año con el manejo alimenticio tradicional de una finca (Veloz, 2008).

La buena alimentación aporta todos los elementos nutritivos que los animales necesitan para compensar los gastos de crecimiento y para mantener un estado sanitario adecuado. La mayoría de los pastos de la región no satisfacen completamente las necesidades nutritivas de los animales debido a que la baja disponibilidad de minerales en el suelo afecta el crecimiento de los forrajes y la acumulación de minerales en sus tejidos (Bedoya, 2012).

Todo animal requiere de adecuadas cantidades de macro y micro elementos esenciales para mantenerse sano y la deficiencia de estos afecta su normal desarro1lo. Los micro elementos esenciales cumplen funciones específicas y decisivas en el metabolismo interno del organismo animal como cofactores específicos de actividad enzimática que ayudan a sintetizar compuestos metabólicos esenciales (Depablos et al., 2009).

La baja disponibilidad de buenos forrajes en la crianza de ganado de carne, especialmente durante la época de sequía, ocasiona un elevado porcentaje de mortalidad en reses adultas y terneros, deficiente desarrollo animal y una marcada reducción de la calidad y productividad bovina (Guzmán, 1988).

Los anabólicos son compuestos que ayudan a la retención nitrógeno $(\mathrm{N})$, elemento indispensable para la síntesis proteica, pero que además favorecen la eritropoyesis (formación de glóbulos rojos) y la retención de calcio $(\mathrm{Ca})$ y fósforo $(\mathrm{P})$, todos éstos, factores que contribuyen a fomentar el aumento de peso de los animales (Loayza, 2012).

Los anabólicos se han utilizado por décadas y actualmente cuentan con el registro y aceptación por parte de la Organización de Alimentos y Agricultura (FAO), la Organización Mundial de la Salud (OMS) y la Agencia de Drogas y Alimentos (FDA), entidades que avalan la legalidad de la práctica en producción de carne bovina porque promueven el crecimiento y no generan riesgos para la salud pública.

Los aditivos modificadores del metabolismo son hormonas que se caracterizan por su función anabolizante, es decir, estimulan la síntesis proteica lo que incrementa la velocidad de crecimiento y la calidad de la canal de los animales de abasto por lo que se han utilizado tradicionalmente en la crianza de rumiantes (Villena \& Jiménez, 2002).

Por otro lado, la utilización de bloques multinutricionales busca eliminar la deficiencia de los nutrientes esenciales que se presenta en los rebaños criados bajo pastoreo o alimentados con residuos de cosechas (Depablos et al., 2009).

La investigación conducida en este tema ha generado resultados importantes que, sin embargo, no se ha enfocado en la ganancia de peso y en el aumento de masa muscular de los animales. El objetivo de esta investigación fue evaluar el efecto de los estimulantes hormonales junto a suplementos minerales y bloques multinutricionales en el engorde de toretes Brahman, combinación que podría reducir el tiempo de crecimiento y engorde en el ganado de carne.

\section{Materiales y métodos}

La presente investigación se realizó en la finca Guayabal con una superficie total de 34 ha, de las cuales 20 están ocupadas en $92 \%$ por pasto miel (Setaria $s p$.) y aproximadamente $8 \%$ por gramíneas invasoras y otros pastos, particularmente gramalote (Paspalum fasciculatum), brachiaria (Brachiaria sp.) y Saboya (Panicum máximum). Las pasturas anteriormente mencionadas han persistido en el potrero durante un tiempo aproximado de 22 años.

El factor en estudio consistió en la utilización de anabólicos sintéticos inyectables combinados con bloques multinutricionales proteicos energéticos mineralizados y vitaminizados. Se consideraron los siguientes tratamientos:

- t0: Alimentación general de la finca con pasto miel + agua + mezcla mineral (testigo absoluto). 
- $\quad \mathrm{t} 1$ : Alimentación general de la finca con pasto miel + bloque multinutricional proteico energético, mineralizado y vitaminizado + anabólico sintético inyectable "a" (undecilenato boldenona) + agua.

- $\quad$ 2: Alimentación general de la finca con pasto miel + bloque multinutricional proteico energético mineralizado y vitaminizado + anabólico sintético inyectable "b" (fenilpropionato nandrolona) + agua.

Para el análisis estadístico se utilizó un diseño completamente al azar con tres tratamientos y seis unidades experimentales por tratamiento. Los animales destinados para la investigación fueron previamente pesados y balanceados con base en su peso corporal y edad antes de ubicarlos en cada grupo a ser evaluado. Las variables evaluadas fueron: incremento de peso, incremento de cinchera, altura a la cruz e incidencia de parásitos externos. La unidad experimental de la investigación estuvo constituida por un torete del grupo racial Brahman mestizo de entre 14 y 15 meses de edad y con un peso vivo entre 250 y 300 kilogramos.

\section{Resultados y discusión}

\subsection{Incremento de peso}

El análisis de la varianza de los datos de incremento de peso indicó que existieron diferencias altamente significativas entre tratamientos. El análisis funcional (Tabla 1) detectó que el mejor tratamiento fue t1 (boldenona undecilenato más bloque multinutricional proteico-energético, mineralizado y vitaminizado) con un incremento de peso promedio de 1,07 $\mathrm{kg} / \mathrm{animal} / \mathrm{día}$, que no fue diferente del tratamiento T2 (fenilpropionato nandrolona más bloque multinutricional proteico-energético, mineralizado y vitaminizado), pero que fue diferente estadísticamente del testigo absoluto con $0,49 \mathrm{~kg} / \mathrm{animal} /$ día en promedio.

\subsection{Incremento de cinchera}

El análisis de la varianza de esta variable también demostró que existieron diferencias altamente significativas entre tratamientos. Las pruebas de análisis funcional detectaron que existió el mismo comportamiento que con incremento de peso cuando se evaluó el incremento del tamaño de la cinchera (Tabla 1). El tratamiento t1 fue el mejor con $0,23 \mathrm{~cm} /$ animal/día, igual que el tratamiento t2, mientras que el testigo solamente alcanzó 0,13 $\mathrm{cm} /$ animal/día. Este comportamiento se debe probablemente a que una de las mayores ventajas del $\mathrm{BM}$ es que ayuda a mejorar el ambiente ruminal del animal al incrementar el número de microorganismos y mejora la relación proteína-energía ayudando de esta forma a la nutrición del animal (McDowel, 1984).

\subsection{Altura a la cruz}

De igual manera, el análisis de la varianza de los datos de incremento de altura a la cruz encontró que se presentaron diferencias altamente significativas entre tratamientos. El análisis de las respuestas para esta variable detectó también dos rangos de significancia, el primero constituido por t1 y t2 con una altura a la cruz de 0,26 y $0,23 \mathrm{~cm} /$ animal/día, respectivamente y el segundo rango claramente diferente constituido por el testigo con $0,10 \mathrm{~cm} /$ animal/día promedio.

Las respuestas observadas con la utilización de implantes hormonales en los parámetros evaluados demuestran que éstos son efectivos para incrementar la tasa de ganancia de peso y el consumo de alimento y para mejorar la eficiencia alimenticia produciendo canales más pesados, con mayor cantidad de músculo y menos grasa. Por ende, el uso de estimulantes hormonales ayuda al desarrollo eficiente de los bovinos y reduce los costos de producción (Ortez, 2012).

Tabla 1. Efecto de los tratamientos en el incremento de peso, cinchera y altura de toretes Brahman mestizos.

\begin{tabular}{lccc}
\hline \multicolumn{1}{c}{ Tratamientos } & $\begin{array}{c}\text { Incremento peso } \\
\mathbf{k g} / \text { animal/día }\end{array}$ & $\begin{array}{c}\text { Incremento cinchera } \\
\mathbf{c m} / \mathbf{a n i m a l} / \mathbf{d i ́ a}\end{array}$ & $\begin{array}{c}\text { Incremento altura } \\
\mathbf{c m} / \text { animal/día }\end{array}$ \\
\hline $\mathrm{t} 1$ = Boldenona Undecilenato $+\mathrm{BM}$ & $1,07 \mathrm{a}^{*}$ & $0,23 \mathrm{a}$ & $0,26 \mathrm{a}$ \\
$\mathrm{t} 2=$ Nandrolona Fenilpropionato $+\mathrm{BM}$ & $0,88 \mathrm{ab}$ & $0,22 \mathrm{ab}$ & $0,23 \mathrm{a}$ \\
$\mathrm{t} 0$ = Testigo Absoluto & $0,49 \mathrm{~b}$ & $0,13 \mathrm{~b}$ & $0,10 \mathrm{~b}$ \\
\hline
\end{tabular}

* Valores con diferente letra difieren estadísticamente según la prueba de Tukey al 5\%. 


\section{Conclusiones}

Los resultados obtenidos en esta investigación sugieren que el tratamiento que produjo la mejor ganancia en peso, en el menor tiempo, fue la utilización de Boldenona Undecilenato más bloque multinutricional proteico energético mineralizado y vitaminizado que permitió un incremento de peso de $1,07 \mathrm{~kg} /$ animal/día, incremento de cinchera de $0,23 \mathrm{~cm} /$ animal/día e incremento de altu-

\section{Referencias}

Bedoya, D. (2012). Evaluación de las relaciones biológicas y económicas entre la producción de las pasturas y la producción lechera del rebaño lactante. Tumbaco, Pichincha. Tesis de pregrado. Facultad de Ciencias Agrícolas. Universidad Central del Ecuador, Quito, Ecuador.

Depablos, L., Ordóñez, J., Godoy, S. \& Chicco, C. (2009). Suplementación mineral proteica de novillas a pastoreo en los Llanos Centrales de Venezuela. Zootecnia Tropical, 27, 249-262.

Guzmán, J. (1988). Pastos y forrajes de Venezuela: producción y aprovechamiento. $2^{\mathrm{da}}$ Ed. Espasande, S.R.L. Caracas, Venezuela.

Loayza, E. (2012). Evaluación del efecto de los anabólicos: zeranol y boldenona en toretes Brahman mestizos alimentados con pasto saboya (Panicum máximum). Piñas, El Oro. Tesis de pregrado. Facultad de Zootecnia. Escuela Superior Politécnica del Chimborazo, Riobamba, Ecuador. ra a la cruz de $0,26 \mathrm{~cm} /$ animal/día. Los datos del análisis económico (no presentado) permiten establecer que el mismo tratamiento tuvo una relación beneficio costo de 1.112 US\$, es decir, por cada dólar invertido se recuperó el dólar y la ganancia marginal neta es de 11 ctvs., en un período de 115 días. El grupo testigo (manejo tradicional) obtuvo incrementos mucho más bajos en peso $(0,49 \mathrm{~kg} /$ animal/día, cinchera $(0,13 \mathrm{~cm} /$ animal/día y altura a la cruz $(0,10 \mathrm{~cm} /$ animal $/ /$ día.

McDowel, J. (1984). Minerales para rumiantes en pastoreo en regiones tropicales. USDA. Departamento de Ciencia Animal, Washington, USA.

Ortez, T. (2012). Ganancia diaria de peso en novillos tratados con dos tipos de implantes anabólicos y alimentados con caña de azúcar. Tesis de pregrado. Departamento de Ciencia y Producción Agropecuaria. Zamorano, Tegucigalpa, Honduras.

Velóz, J. (2008). Evaluación de la suplementación proteico-energético en la alimentación de vaconas Fierro Holstein Friesian. CADET, Tumbaco, Pichincha. Tesis de pregrado. Facultad de Ciencias Agrícolas. Universidad Central del Ecuador, Quito, Ecuador.

Villena, E. \& Jiménez, J. (2002). Técnico en ganadería. Eitorial Cultural, Madrid, España 